\title{
American College of Medical Genetics and Genomics: standards and guidelines for documenting suspected consanguinity as an incidental finding of genomic testing
}

\author{
Catherine W. Rehder, PhD ${ }^{1}$, Karen L. David, MD, MS'2,3, Betsy Hirsch, PhD ${ }^{4}$, Helga V. Toriello, PhD \\ Carolyn M. Wilson, $\mathrm{MS}^{6}$ and Hutton M. Kearney, $\mathrm{PhD}^{6}$
}

Genomic testing, including single-nucleotide polymorphism-based microarrays and whole-genome sequencing, can detect long stretches of the genome that display homozygosity. The presence of these segments, when distributed across multiple chromosomes, can indicate a familial relationship between the proband's parents. This article describes the detection of possible consanguinity by genomic testing and the factors confounding the inference of a specific parental

Many genomic microarray platforms use a combination of probes designed to assess copy number and probes to genotype single-nucleotide polymorphisms. In addition to copy-number changes (i.e., deletions, duplications), these array platforms can identify genomic regions that display an absence of heterozygosity, often in the form of one or more long contiguous stretch of homozygosity. Large regions of homozygosity, when observed on a single chromosome, can be indicative of uniparental disomy; ${ }^{1}$ however, when these regions are distributed throughout the genome, they usually represent segments of autozygosity or regions that are identical by descent (IBD). These autozygous segments originate from a common ancestor and can indicate a consanguineous relationship between the proband's parents. The health impact of consanguinity has been recently reviewed. ${ }^{2}$ Whole-genome and potentially wholeexome sequencing strategies can also detect long contiguous stretches of homozygosity. Because the results obtained using any of these technologies can point to a familial relationship or consanguinity between parents, these technologies could reveal situations suspicious for potential abuse, especially, but not limited to, situations when the mother is disabled or a minor. ${ }^{3}$

The guidelines presented here are designed to assist clinical laboratories in the management of microarray and exome/ genome sequencing findings that suggest parental consanguinity, with a primary focus on detection and reporting results back to the ordering clinician.

\section{DETECTION OF CONSANGUINITY}

Genomic regions that are IBD originate from a common ancestor, with the proportion of the genome that is autozygous relationship. It is designed to guide the documentation of suspected consanguinity by clinical laboratory professionals and to alert laboratories to the need to establish a reporting policy in conjunction with their ethics review committee and legal counsel.

Genet Med 2013:15(2):150-152

Key Words: consanguinity; homozygosity; laboratory guideline

increasing as the parental relationship becomes closer. The average proportion of the autosomal genome that is IBD in the offspring of related parents is given by the coefficient of inbreeding $(F) .{ }^{4}$ For example, on average, $6.25 \%$ or $1 / 16$ th of the genome of offspring of first cousins $(F=1 / 16)$ is IBD. Although the coefficient of inbreeding provides a theoretical value, significant deviations from the expected values do occur.

Because smaller stretches of homozygosity $(<3 \mathrm{Mb})$ spread throughout the genome are common even in outbred populations, laboratories typically set a size threshold, below which segments are not considered significant. In theory, in the offspring of a second-cousin mating, an average of four $12.5 \mathrm{Mb}$ stretches of homozygosity per genome will be present, although both the number and the size of homozygous segments are known to be highly variable. ${ }^{5}$ When long contiguous stretches of homozygosity involving multiple chromosomes are present, the percentage of the genome that is IBD can be estimated by the sum of the sizes of the homozygous segments divided by the total autosomal genomic length ( 2,881 Mb for GRCh37/hg19). The sex chromosomes are typically excluded from the calculation because males have only a single $\mathrm{X}$ and $\mathrm{Y}$ chromosome and therefore cannot have homozygosity at any locus outside of the pseudoautosomal regions. This calculation is likely an underestimation of the actual percentage of the genome that is IBD because only those segments of homozygosity meeting the threshold set by the laboratory will be flagged for inclusion in the calculation. ${ }^{6}$ This percentage can then be compared with the theoretical value derived from the coefficient of inbreeding for any given parental relationship. These theoretical values are found in many genetics texts and resources. ${ }^{5}$

${ }^{1}$ Duke University Health System, Durham, North Carolina; ${ }^{2}$ Metropolitan Hospital Center, New York, New York; ${ }^{3}$ New York Methodist Hospital, Brooklyn, New York; ${ }^{4}$ University of Minnesota Medical School, Minneapolis, Minnesota; ${ }^{5}$ Spectrum Health, Grand Rapids, Michigan; ${ }^{6}$ Fullerton Genetics Center, Mission Health, Asheville, North Carolina. Correspondence: Catherine W. Rehder (catherine.rehder@duke.edu) 
Because recombination during meiosis is a somewhat random process, the variation from the theoretical value increases with each meiosis ${ }^{7}$ such that third cousins may share more DNA sequences than second cousins. Even among the progeny of first cousins, in whom the average percentage of the genome that is IBD is $6.25 \%$, the SD is $2.43 \% .^{5}$ The expected percentages are based on a single common ancestor; however, multiple loops of consanguinity or multiple generations of breeding within a relatively closed community could complicate the estimation of the degree of relationship. These variations from the expected or theoretical values are more pronounced for more distantly related individuals ${ }^{5}$ and may be caused by stochastic events, multiple loops of consanguinity, small gene pools, and unknown family structures (e.g., adoptions, nonpaternity). Because of these variables, the specific familial relationship or degree of relatedness between the parents cannot always be extrapolated from the percentage of the genome that is IBD. Single-nucleotide polymorphism array analysis is not designed to be a paternity test, nor should it be used to definitively assign a specific relationship between the parents of the proband.

Concerns for abuse arise when IBD proportions suggest that the parents of the proband are first- or second-degree relatives, particularly when the mother is a minor or intellectually disabled. Among the progeny of first- $(F=1 / 4)$ and second-degree $(F=1 / 8)$ relative matings, the number of meioses separating the parents is sufficiently low such that the SD is relatively low. Therefore, when high percentages of the genome $(\geq 10 \%)$ are IBD and several large segments of absence of heterozygosity are present, it is reasonable to suspect a close parental relationship.

\section{RECOMMENDATIONS FOR PRETEST COUNSELING}

It is recommended that each patient/family undergoing microarray and exome/genome testing receive pretest counseling.

\section{RECOMMENDATIONS FOR REPORTING FINDINGS OF CONSANGUINITY TO THE ORDERING CLINICIAN}

It is important to recognize that the detection of one or more long contiguous stretch of homozygosity, in and of itself, is not abnormal. However, the detection of segments that are homozygous does increase the likelihood that the proband has inherited two copies of a deleterious allele for an autosomal recessive disorder. Clinicians may find utility in this knowledge if the patient's phenotype matches that of an autosomal recessive disorder for which one or more candidate genes are located within one of these segments. Because there is clinical utility in the detection of excessive homozygosity, even when the percentage of the genome that is IBD is quite low $(<3 \%)$, many laboratories may choose to report this finding back to the ordering clinician to encourage consideration of recessive mechanisms and facilitate autozygosity mapping. Laboratories may choose to include a percentage or proportion of the genome that is homozygous in their reports. In general, caution should be exercised when using an automated calculation of the percentage of the genome that is IBD. Some analysis programs generate this calculation using all segments displaying absence of heterozygosity, regardless of size or mechanism, which can include deletions. This automated calculation is also typically inflated by small regions of homozygosity that are more likely representative of regions of suppressed recombination or linkage disequilibrium (identity by state). Limiting this calculation to segments $>2-5 \mathrm{Mb}$ is more likely to result in the inclusion of segments that are truly IBD. Each laboratory should establish parameters for calculating the percentage of the genome that is IBD and determine a threshold for reporting back the results.

In general, laboratories have very limited information regarding the structure of the proband's family (e.g., maternal age, adoptions, multiple loops of consanguinity, other familial relationships). Therefore, speculation of a specific relationship in written reports is strongly discouraged. An example of suggested language is as follows:

"Several large regions of homozygosity (_ Mb or larger) were detected, encompassing >_\% of the genome. Although this result is not diagnostic of a specific condition, it raises the possibility of a recessive disorder with a causative gene located within one of these regions. A genetics consultation is recommended."

\section{SPECIAL CONSIDERATIONS}

The observation of a possible first- or second-degree parental relationship, particularly when the mother of the proband is known to be a minor or has an intellectual disability, raises a suspicion for abuse involving the mother of the proband. Laboratories do not typically have information regarding the mother's age, intellectual status, or family structure; therefore, they do not have adequate information to communicate a suspicion for abuse to any authoritative agency. Therefore, when the percentage of homozygosity reaches a level that could be consistent with a first- or second-degree parental relationship (>10\% with multiple regions of homozygosity $2-5 \mathrm{Mb}$ or larger), laboratory reports should indicate that the results could be associated with possible consanguinity to ensure that the ordering clinician (geneticist or nongeneticist) understands the implications of the results. An example of suggested language is as follows:

"Several large regions of homozygosity (_ Mb or larger) were detected, encompassing >_\% of the genome. Although this result is not diagnostic of a specific condition, it raises the possibility of a recessive disorder with a causative gene located within one of these regions. Additionally, these results could indicate a familial relationship (first or second degree) between this individual's parents. A genetics consultation is recommended."

Laboratories are encouraged to engage the ordering clinician when a first- or second-degree mating is suspected based on the results of the analysis. The clinician is the most appropriate 
person to correlate laboratory results with family history and cultural traditions and to investigate any concern for abuse. Clinicians should be aware that many states have mandatory reporting statutes requiring that anyone with cause to suspect that a child, juvenile, or disabled adult has been the victim of abuse, including rape or sexual assault, report his/her concern to the appropriate governmental authorities. ${ }^{8,9}$ These same statutes provide protection for the reporting individual as long as the concern is raised in good faith. It is advised that each laboratory or hospital consult with its ethics review committee and legal counsel for policy development concerning the requirements for and manner of reporting.

\section{CONCLUDING REMARKS}

The ability to detect regions of homozygosity is an important clinical tool with clear utility in the context of the detection of autosomal recessive conditions and uniparental disomy. A secondary consequence of the observation of regions of homozygosity is the possible discovery of a consanguineous relationship between the proband's parents. Although a specific relationship cannot be determined using the currently available technologies, this information may be useful to the clinician caring for the patient and family. It is the responsibility of the clinician, not the laboratorian, to perform clinical correlation and investigate any concern for abuse. The laboratorian's duty is to effectively communicate the possibility of a familial relationship between the parents to the ordering clinician when a first- or second-degree relationship is suspected based on the results of the analysis. Laboratories are encouraged to develop a reporting policy in conjunction with their ethics review committee and legal counsel.

\section{ACKNOWLEDGMENTS}

The authors acknowledge Rebecca Anderson for her expert advice in the formation of this guideline and her critique of the manuscript.

\section{DISCLOSURE}

The authors declare no conflict of interest.

\section{REFERENCES}

1. Papenhausen $P$, et al., UPD detection using homozygosity profiling with a SNP genotyping microarray. Am J Med Genet Part A 2011;155A:757-768.

2. Hamamy H, Antonarakis SE, Cavalli-Sforza LL, et al. Consanguineous marriages, pearls and perils: Geneva International Consanguinity Workshop Report. Genet Med 2011;13:841-847.

3. Schaaf CP, Scott DA, Wiszniewska J, Beaudet AL. Identification of incestuous parental relationships by SNP-based DNA microarrays. Lancet 2011;377:555556.

4. Hartl DL, Clark AG. Principles of Population Genetics. Sinauer Associates: Sunderland, MA, 1997.

5. Carothers AD, Rudan I, Kolcic I, et al. Estimating human inbreeding coefficients: comparison of genealogical and marker heterozygosity approaches. Ann Hum Genet 2006;70(Pt 5):666-676.

6. Kearney HM, Kearney JB, Conlin LK. Diagnostic implications of excessive homozygosity detected by SNP-based microarrays: consanguinity, uniparental disomy, and recessive single-gene mutations. Clin Lab Med 2011;31:595613 , ix.

7. McQuillan R, Leutenegger AL, Abdel-Rahman R, et al. Runs of homozygosity in European populations. Am J Hum Genet 2008;83:359-372.

8. Burgess AW. How many red flags does it take? Am J Nurs 2007;107:28-31.

9. West JC. Case law update: a review of recent rulings of interest to risk managers. Anonymous Hospital v A.K., 920 N.E.2d 704 (Ct. App. Ind., January 26, 2010). J Healthc Risk Manag 2010;30:36-37. 